\title{
Effect of inhomogeneities on streamer propagation: II. Streamer dynamics in high pressure humid air with bubbles
}

\author{
Natalia Yu Babaeva and Mark J Kushner ${ }^{1}$ \\ Department of Electrical Engineering and Computer Science, University of Michigan, 1301 Beal Ave., \\ Ann Arbor, MI 48109, USA \\ E-mail: nbabaeva@umich.edu and mjkush@umich.edu
}

Received 26 December 2008, in final form 8 April 2009

Published 29 May 2009

Online at stacks.iop.org/PSST/18/035010

\begin{abstract}
The branching of electric discharge streamers in atmospheric pressure air, dense gases and liquids is a common occurrence whose origins are likely found with many causes, both deterministic and stochastic. One mechanism for streamer branching may be inhomogeneities in the path of a streamer which either divert the streamer (typically a region of lower ionization) or produce a new branch (a region of higher ionization). The propagation and branching of streamers in liquids is likely aided by low density inhomogeneities, bubbles; however, modeling of streamers in liquids is made difficult by the lack of transport coefficients. As a first step towards understanding the propagation and branching of streamers in liquids, we investigated the consequences of random inhomogeneities in the form of low pressure bubbles on the propagation of streamers in high pressure humid air. By virtue of their lower density, bubbles have larger $E / N$ (electric field/gas number density) than the ambient gas with larger rates of ionization. The intersection of a streamer with a bubble will focus the plasma into the bubble by virtue of that higher rate of ionization but the details of the interaction depend on the relative sizes of the bubble and streamer. When a streamer intersects a field of bubbles, the large $E / N$ in the bubble avalanches seed electrons produced by photoionization from the streamer. Each bubble then launches both a negative and positive going streamer that may link with those from adjacent bubbles or the original streamer. The total process then appears as streamer branching.
\end{abstract}

(Some figures in this article are in colour only in the electronic version)

\section{Introduction}

The branching of electric discharge streamers occurs in most high pressure gases and liquids [1,2]. Although many proposals have been made for the source of branching, it is likely that the cause is multifaceted and depends on many parameters, for example the type of gas or liquid, pressure and the rate of voltage rise. Branching is so common that the dominant cause cannot be too system specific although the processes determining the details of branching (e.g. number and length of branches) are likely to be more system specific. This is particularly true for streamers in liquids and high

\footnotetext{
${ }^{1}$ Author to whom any correspondence should be addressed.
}

pressure gases where branching nearly always occurs while the morphology of the branches depends on discharge conditions.

Streamer dynamics in liquids have attracted particular attention due to their intrinsic multiphase nature [1]. That is, discharges in liquids often involve a pre-existing gas phase, such as naturally occuring bubbles, or produce their own gas phase through Joule heating or other means. Discharges in liquids, and water in particular, are of interest because they generate chemically active species (e.g. $\mathrm{OH}, \mathrm{H}_{2} \mathrm{O}_{2}$ ) and so can be used for disinfection and purification of water by removal of volatile organic compounds [3-7] and for the destruction of bacteria $[8,9]$. Electric discharges in bubbles within liquids have also been used as a source of UV radiation, ozone and hydrogen peroxide $[10,11]$. Liquids can have high dielectric 
strength, meaning that they are able to withstand many $\mathrm{kV} \mathrm{cm}^{-1}$ without breakdown and, unlike solid dielectrics, self repair after breakdown. This makes liquids attractive for high voltage insulation [12]. These same properties make liquids attractive as a switching medium [12]. Plasmas in water and saline solutions are of particular interest in the context of tissue treatment and surgery [13].

Electric breakdown of dielectric fluids (including liquids and high pressure gases) is a complex process that involves a succession of inter-correlated phenomena (e.g. electronic, thermal, mechanical) while also depending on experimental conditions (e.g. electrode geometry and shape, applied voltage waveform and liquid nature and purity) [14]. In spite of their very different conditions, discharges in liquids may share some properties with high pressure gases. For example, discharges in liquids are often corona-like, where the plasma is filamentary with many branches which at least visibly is similar to streamers in high pressure gases [14-19].

The mechanism for initiating discharges in liquids is often classified as either electronic or thermal. In a short pulse regime (hundreds nanoseconds or less) discharges in liquids are similar to those in gases in that ionization seemingly occurs directly in the liquid phase by electron impact on the liquid molecules without the production of gas phase. The thermal (or bubble) mechanism is usually associated with longer pulses when heating of the liquid by ionic current enhances the formation of gaseous bubbles. Based purely on analogies to gases, the initiation and propagation of discharges in liquids should require the production of a gas phase. The observed $E / N$ (electric field/neutral number density) in liquids for streamer propagation is typically too low for conventional avalanches to occur based on the gas phase analogues. By the formation of a gas phase, the $E / N$ increases by the ratio of the density of the ambient liquid phase and the gaseous bubble, a factor of hundreds to a few thousand. This produces values of $E / N$ more consistent with breakdown and streamer propagation in dense gases.

There are several mechanisms to explain the existence of bubbles in common liquids during discharges. Bubbles can be formed in the high electric field region at the tip of the electrode. The electric field coupled with the dielectric properties of the liquid can cause volume compression or expansion [1]. Microscopic bubbles can pre-exist in the liquid due to pouring and liquid motion. Bubbles can be formed in the process of the discharge propagation by Joule heating or by the high electric field in the avalanche front of a streamer [20,21]. The electrical breakdown of long vapor bubbles in capillaries generated by the external heating of the capillary was studied by Bruggeman et al [22]. All of these bubbles would typically be of the saturated vapor of the liquid. Bubbles of other composition can, of course, be intentionally injected into the liquid [23].

Although there are many experiments which lend credence to bubble enhanced propagation of streamers in liquids, there are few fundamental theories or models based on first principles which are able to capture the sequence of events from seed electron in liquid to gas phase plasma channel. There have been only a limited number of numerical studies of propagation of streamers in pure liquids due in large part to the lack of transport coefficients. For example, Babaeva and Naidis modeled streamers in liquid Ar using gas-modified transport coefficients which addressed the electronic mechanism of streamer propagation [24]. They found that unlike gas phase streamers, electron-ion recombination is an important process in the liquid phase. The most relevant simulations to date have been performed by Qian et al who investigated, using Monte Carlo techniques, the enhanced ionization that may occur in micro-bubbles as electrons transport through water [25].

To lend insights to how bubbles might enhance propagation of streamers in liquids and possibly produce branching, in this paper we report on results from a computational investigation the role of randomly distributed regions of low density (i.e. bubbles) in the propagation and branching of a streamer in high pressure humid air $\left(\mathrm{N}_{2} / \mathrm{O}_{2} / \mathrm{H}_{2} \mathrm{O}=79 / 20 / 1\right)$. The authors acknowledge that there are important additional phenomena that would be necessary to include in modeling streamer propagation through a liquid with bubbles. For, example the high permittivity of water $\left(\varepsilon / \varepsilon_{0}=\right.$ 80 ) would produce bending of electric field lines at the interface with the bubble and electric field enhancement. The finite conductivity and low mobility of charge carriers in the liquid would allow for charge buildup at the gas-liquid interface. The larger ratio of densities between the bubbles and ambient liquid would produce even larger values of $E / N$ in the bubble than discussed here. The absorption of photoionizing radiation in intervening liquid would be proportionately larger than in dense air. Nevertheless, this study provides a well controlled environment to investigate some of the basic properties of streamer propagation through multiple phases.

In this study, we extend our investigation reported on in Part I [26] to streamer interactions with a single bubble and with fields of bubbles having a large degree of rarefaction. In our simulations the streamer propagates between (or simultaneously) through two gas regions, dense and rarefied, which may approximate the liquid (ambient) and gas (bubbles) phases during the initial breakdown of a liquid dielectric. The approximate nature of this calculation and the means to generate the bubbles are discussed in Part I.

We found that rarefied regions, bubbles, can enhance the propagation of streamers in dense media through the elevated $E / N$ in those regions. The seeding of electrons by photoionization in these regions initiates avalanches which produce positive (cathode directed) and negative (anode directed) streamers. Depending on the size, pressure difference and distribution of the bubbles, the streamers may branch or launch new streamers which collectively provide the appearance of branching. The fact that the bubbles are stochastically distributed in the dense phase leads to random branching.

The model used in this study is described section 2 . Typical streamer characteristics in the absence of bubbles are discussed in section 3 . The consequences of streamers interacting with a single bubble on the axis and multiple randomly distributed bubbles are discussed in sections 4 and 5 . The roles of bubble size and internal pressure are discussed in section 6 . The dependence of streamer branching on the mean free path for photoionizing radiation is discussed in section 7 . Concluding remarks are in section 8. 

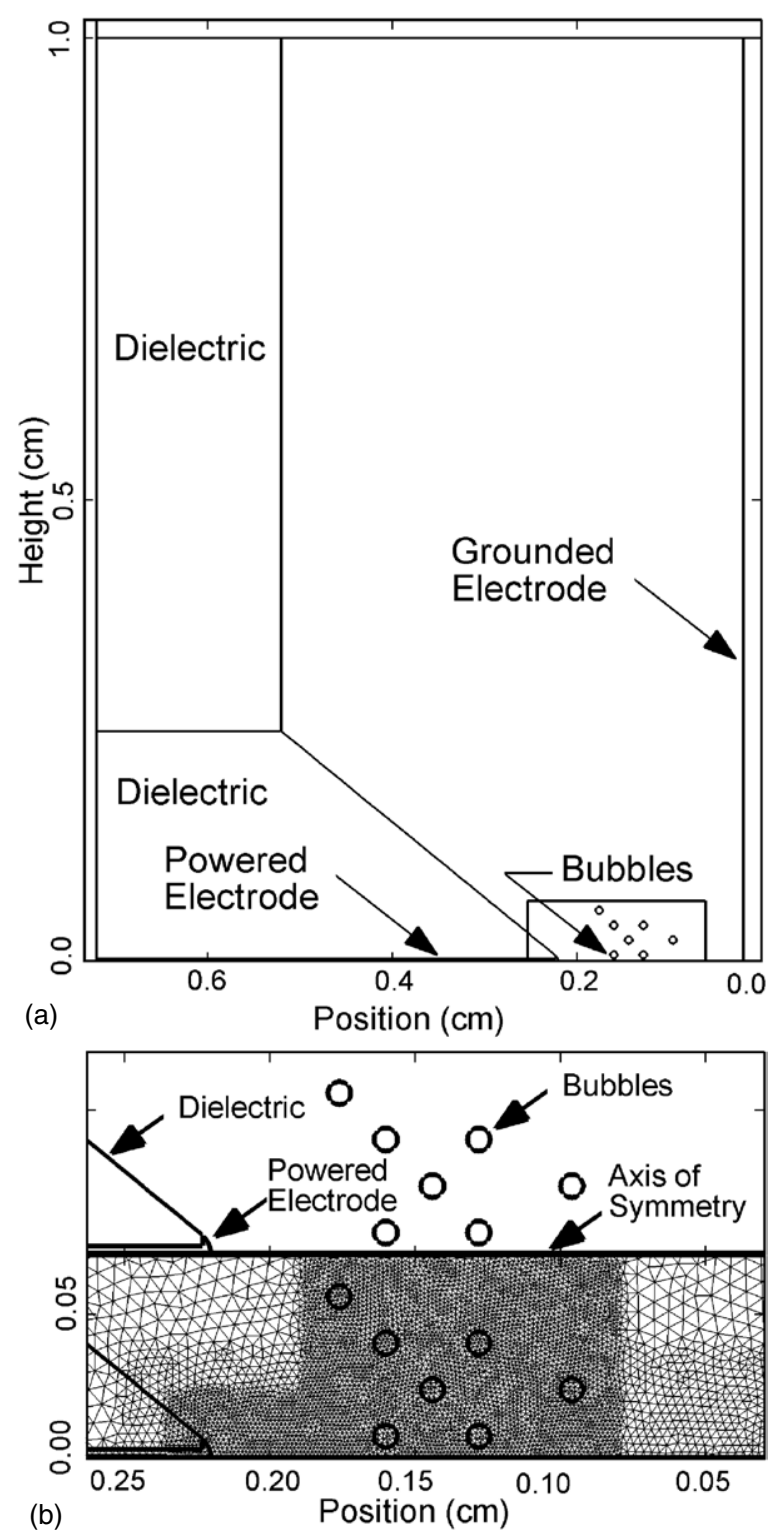

Figure 1. Schematic of the geometry. (a) Entire computational domain, $(b)$ close up of the anode-cathode gap. Typical numerical mesh is shown to resolve seven bubbles.

\section{Description of the model}

The model, nonPDPSIM, and the reaction mechanism used in this study are the same as those described in Part I [26]. and so will be only briefly described here. nonPDPSIM is a two-dimensional simulation performed on an unstructured mesh in which Poisson's equation for the electric potential and transport equations for charged and neutral species are solved. The electron temperature, $T_{\mathrm{e}}$, is obtained by solving an electron energy conservation equation with transport and rate coefficients coming from local solutions of Boltzmann's equation. Radiation transport and photoionization are included by implementing Green's function propagator. The model geometry is shown in figure $1(a)$. A positive corona discharge is sustained between a rod encased in a dielectric $\left(\varepsilon / \varepsilon_{0}=5\right)$ having an exposed edge with a radius of curvature of $0.07 \mathrm{~cm}$ and a ground plane $2 \mathrm{~mm}$ away. The geometry is symmetric across the center line.
The numerical grid uses an unstructured mesh with triangular elements with refinement regions to resolve the details of the electrode tip and the bubbles, as well as the larger surrounding features. The schematic shown in figure $1(b)$ is the mesh used for a case with seven randomly distributed bubbles. The mesh consists of approximately 7700 nodes, of which about 6300 are in the plasma region. The mesh resolution is 1-4 $\mu \mathrm{m}$ near the anode and bubbles. The resolution of the mesh was varied to confirm that the predicted streamer properties do not significantly change based on the mesh size.

Unless otherwise noted, the gas mixture is $\mathrm{N}_{2} / \mathrm{O}_{2} / \mathrm{H}_{2} \mathrm{O}=$ $79 / 20 / 1$ with an ambient pressure of $P_{0}=3 \mathrm{~atm}$. The bubble radii we investigated were 10 to $320 \mu \mathrm{m}$ with pressures, $P$, of $0.2 \mathrm{~atm}\left(P / P_{0}=0.065\right)$ to $1 \mathrm{~atm}\left(P / P_{0}=0.33\right)$. For the majority of cases discussed here the mean free path of photoionizing photons was $750 \mu \mathrm{m}$.

\section{Streamers in the absence of bubbles}

The properties of a streamer propagating through $3 \mathrm{~atm}$ humid air in the absence of bubbles are shown in figure $2(E / N$, electron density and net positive charge) and figure $3\left(T_{\mathrm{e}}\right.$, positive electron impact source and photoionization source). The streamer has the same general structure as that for a streamer in humid air at $1 \mathrm{~atm}$ as shown in figure 2 of Part I [26]. A potential of $30 \mathrm{kV}$ with a rise time of $0.1 \mathrm{~ns}$ was applied to the electrode. In the absence of bubbles, a single streamer propagates from the electrode tip to the ground plane. (For reference, the outlines of the bubbles to be placed in a subsequent case are shown.) The positive space charge shown in figure 2(c) outlines the streamer and so can be used to determine its width. The maximum space charge is at the streamer tip $\left(4 \times 10^{13} \mathrm{~cm}^{-3}\right)$ which sustains an $E / N$ as large as $610 \mathrm{Td}\left(1 \mathrm{Td}=10^{-17} \mathrm{~V} \mathrm{~cm}^{2}\right)$ or $450 \mathrm{kV} \mathrm{cm}^{-1}$ at $3 \mathrm{~atm}$. This produces $T_{\mathrm{e}}$ of up to $5 \mathrm{eV}$ in the avalanche front. The charge density at the sides of the streamer channel $\left(1 \times 10^{13} \mathrm{~cm}^{-3}\right)$ provides the confining ambipolar electric fields $\left(E / N=150 \mathrm{Td}\right.$ or $\left.110 \mathrm{kV} \mathrm{cm}^{-1}\right)$ which prevent the streamer from spreading laterally.

Trailing the avalanche front, the conducting channel of the streamer has a lower $E / N$ of only a few tens of $\mathrm{Td}$ and $T_{\mathrm{e}}<1-2 \mathrm{eV}$. Photoionization from the avalanche front and plasma channel producs electrons up to a few hundred micrometers in front of the streamer front. As these electrons drift into the large $E / N$ of the streamer front, they avalanche in the region of large $T_{\mathrm{e}}$ to propagate the streamer, producing electron impact sources of $2 \times 10^{23} \mathrm{~cm}^{-3} \mathrm{~s}^{-1}$ early during the streamer development and $3 \times 10^{24} \mathrm{~cm}^{-3} \mathrm{~s}^{-1}$ later. Note that only the positive sources are shown in figure 3 there is a net attachment in the conducting streamer column immediately after the avalanche front. Photoionization sources can, in fact, dominate in the conducting column at this time due to the finite lifetimes of highly excited states and the low $T_{\mathrm{e}}$.

As the conducting channel occupies a larger fraction of the inter-electrode gap, a larger fraction of the applied voltage is compressed into the smaller gap ahead of the streamer. The result is that the $E / N$ in the streamer tip and electron 
(a)

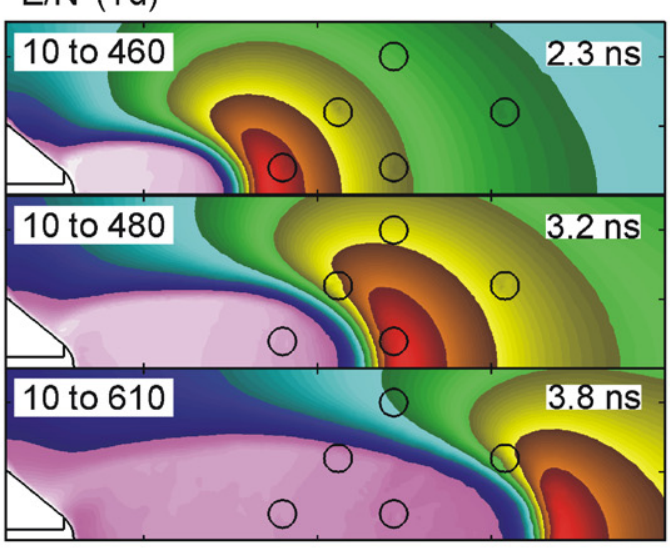

Electron density $\left(\mathrm{cm}^{-3}(2 \mathrm{dec})\right)$

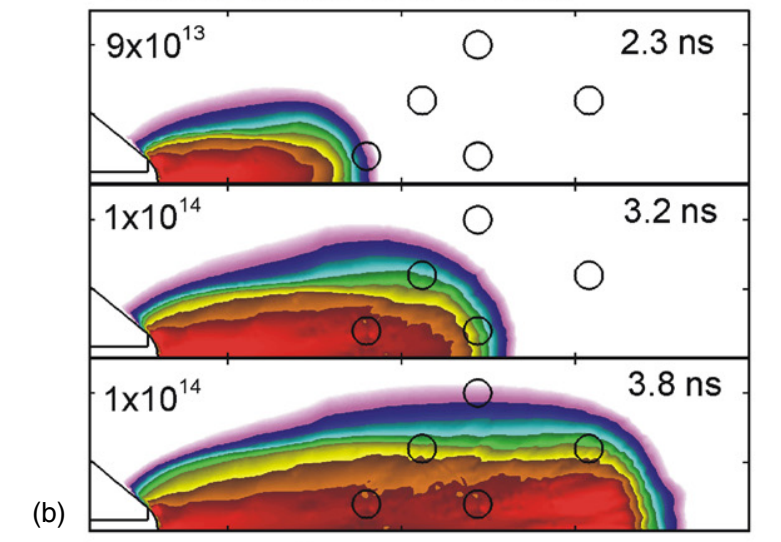

(b)

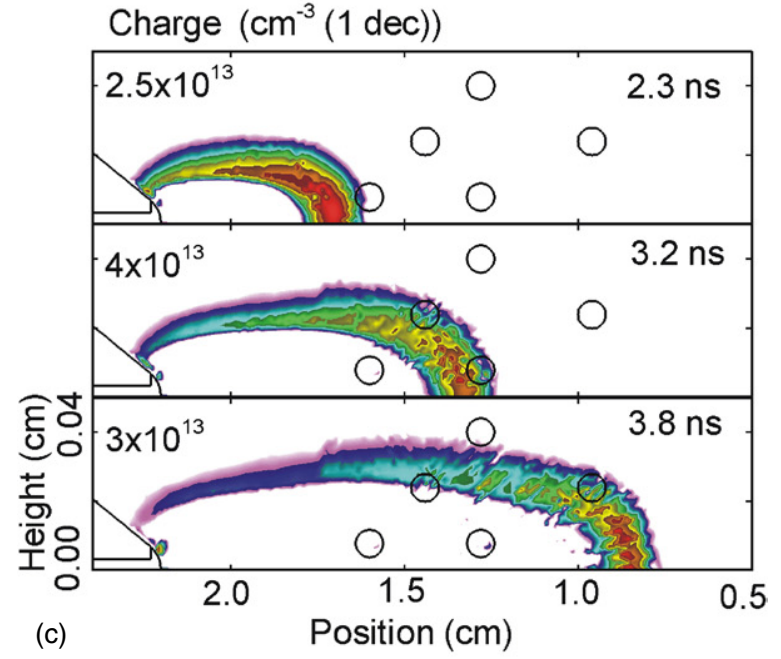

MIN

Figure 2. Streamer properties in 3 atm of humid air without bubbles. (The outlines of bubbles are shown for reference to later results.) (a) Reduced electric field $E / N$. (b) electron density and $(c)$ net positive charge. The range of values plotted or maximum value is shown in each frame. Plots are on a log scale if number of decades is noted. In the absence of bubbles, the streamer propagates as a single filament.

impact ionization sources increase. This in turn increases the propagation speed of the streamer. Typically the streamer width is proportional to the applied voltage and inversely proportional to pressure $[27,28]$. It can also depend on the mean free path of photoionizing radiation $\lambda_{\mathrm{P}}$. As discussed in (a)

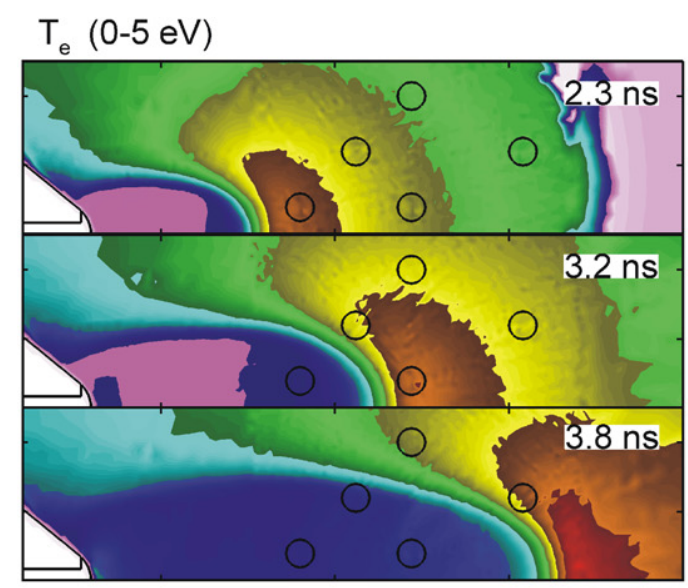

\section{E-Impact Source $\left(\mathrm{cm}^{-3} \mathrm{~s}^{-1}(2 \mathrm{dec})\right)$}

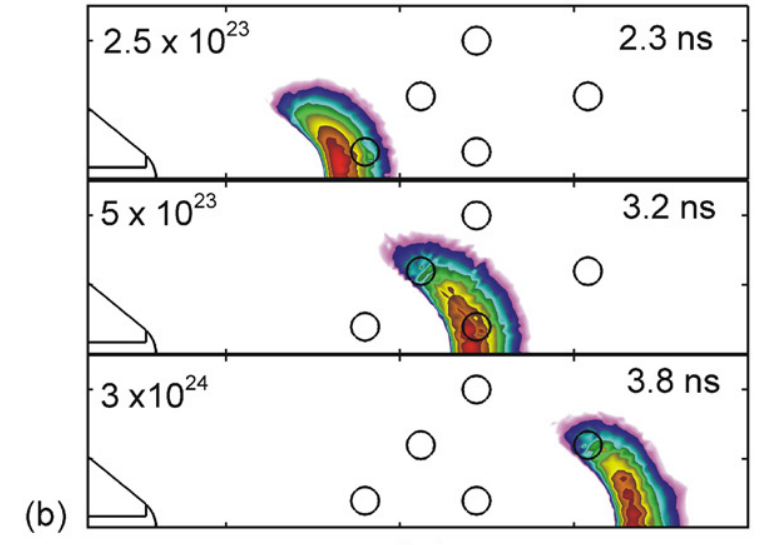

Photo Source $\left(\mathrm{cm}^{-3} \mathrm{~s}^{-1}(2 \mathrm{dec})\right)$

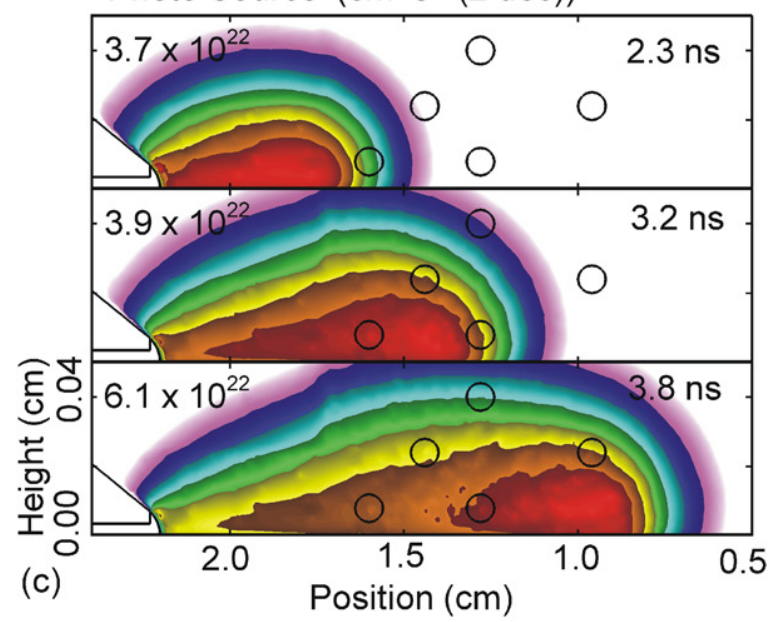

MIN

Figure 3. Streamer properties in $3 \mathrm{~atm}$ of humid air without bubbles. (The outlines of bubbles are shown for reference to later results.) (a) Electron temperature $T_{\mathrm{e}},(b)$ positive electron impact ionization source and $(c)$ photoionization source. The range of values plotted or maximum value is shown in each frame. Plots are on a $\log$ scale if number of decades is noted.

section 7 , the streamer becomes thicker with increasing $\lambda_{P}$ as seed electrons are more widely distributed.

Although extensive parametrizations have not been performed, the trends we discuss here and below are independent of the amount of water vapor up to $10-20 \%$. The 
most sensitive parameter when changing the humidity is the change in the propagation of photoionizing radiation.

\section{Streamer intersection a single rarefied bubble on axis}

Streamer dynamics, morphology and propagation speed can be significantly perturbed by an inhomogeneity (or inhomogeneities) in its path. In Part I we investigated inhomogenieties in the form of bubbles having positive and negative pressure perturbations where $\Delta P / P_{0}$ did not exceed $7-8 \%$. These less dramatic changes in streamer properties provided a baseline for how inhomgeneities can affect streamer propagation. To make closer analogies to the effects of bubbles in liquids, here we discuss the role of inhomogeneities having greater rarefaction with $P / P_{0}=0.33,0.2,0.065$ in an ambient pressure $P_{0}=3 \mathrm{~atm}$.

The intersection and propagation of streamers through bubbles depends on the relative dimensions of the streamer and the bubbles, $P / P_{0}$, the photoionization mean free path, $\lambda_{\mathrm{P}}$, and the number and spatial distribution of the bubbles. To set a baseline, we first discuss the intersection of a streamer with a single bubble of $320 \mu \mathrm{m}$ radius (larger than the streamer width) on the streamer axis with $P / P_{0}=0.065$ ( $P=150$ Torr, $P_{0}=$ $3 \mathrm{~atm}$ ). Streamer properties are shown in figure 4 (electron density, net negative charge and net positive charge) and figure $5(E / N$, electron impact sources and photoionization sources).

The streamer initially develops from the tip of the electrode in the conventional way by launching a wave of positive space charge towards the cathode forming a positive cathode directed streamer (CDS). The ambient $E / N$ is near $150 \mathrm{Td}$ whereas the $E / N$ in the bubble is $3000 \mathrm{Td}$. Photoionization from the streamer seeds electrons in front of the main streamer channel and so sustains the streamer. The photon mean free path is long enough that a small density of electrons is seeded in the bubble in advance of the streamer's arrival. The rate of photoionization is roughly in proportion to the absorbing gas density or about 15 times smaller in the bubble than in the ambient. In spite of the lower rate of electron generation in the bubble compared with the surrounding ambient, the elevated $E / N$ in the bubble launches a cathode directed ionization wave in the bubble. This wave is indicated by both the ionization source and the positive space charge. The $E / N$ in the ionization wave in the bubble increases to $4500 \mathrm{Td}$. Electron densities exceeding $6 \times 10^{14} \mathrm{~cm}^{-3}$ are produced in the bubble, about 6 times larger than in the absence of the bubble. The plasma inside the bubble occupies nearly its entire volume, which is consistent with the width of the streamer being inversely proportional to pressure [27, 28].

At this time, the primary streamer from the electrode tip has yet to reach the bubble. Electrons produced in the bubble drift towards the anode, cross the bubble boundary and initiate a negative anode directed streamer (ADS) as indicated by the negative space charge. Now there are three streamers- the CDS in the bubble and from the electrode tip and the ADS from the left side of the bubble. The CDS from the electrode and the ADS intersect, after which there is a significant acceleration of
$P / P_{0}=0.065$

Electron density $\left(\mathrm{cm}^{-3}(3 \mathrm{dec})\right)$

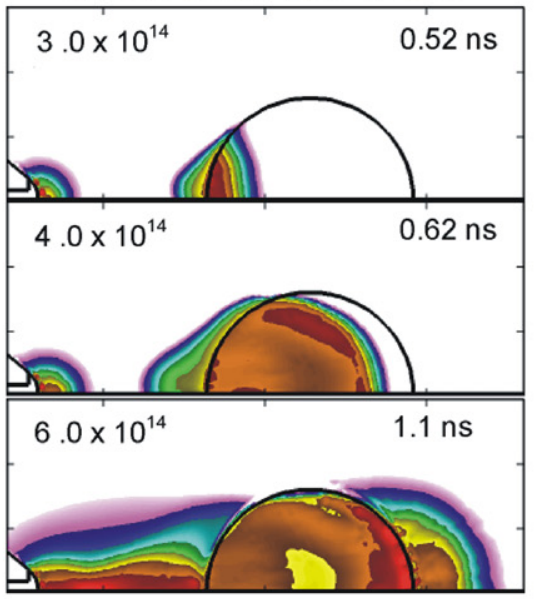

Negative charge $\left(\mathrm{cm}^{-3}(2 \mathrm{dec})\right)$

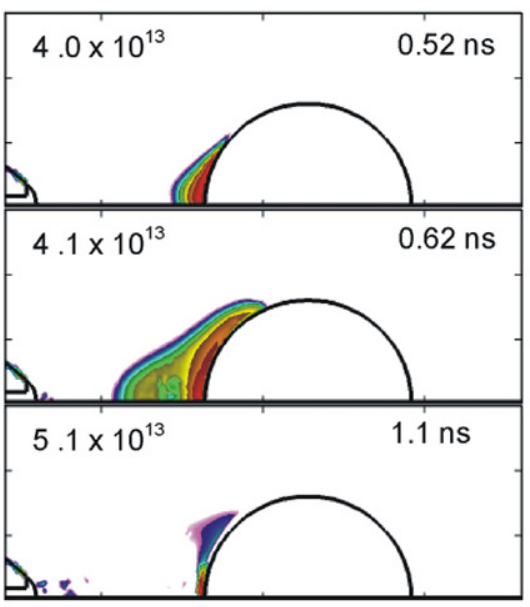

Positive charge $\left(\mathrm{cm}^{-3}(1 \mathrm{dec})\right)$

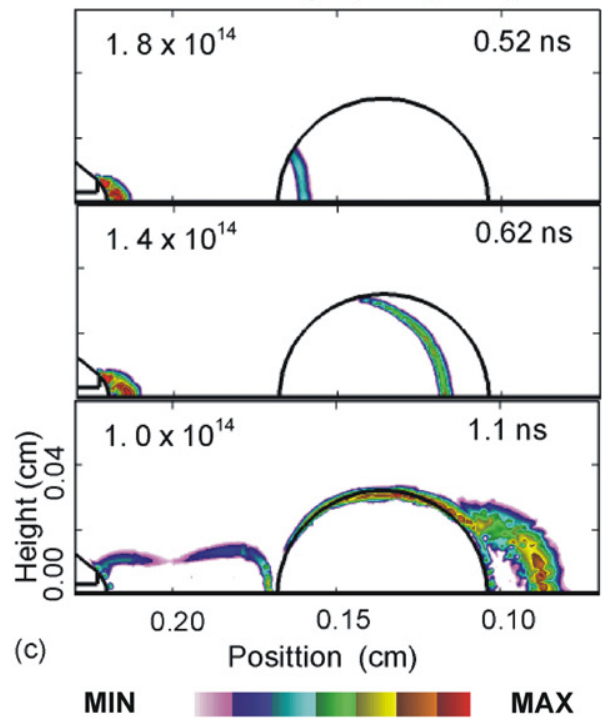

Figure 4. Streamer intersecting a single bubble of $320 \mu \mathrm{m}$ radius located on axis for ambient pressure of $3 \mathrm{~atm}$ and $P / P_{0}=0.065$. $(a)$ Electron density, $(b)$ net negative charge and $(c)$ net positive charge. There is a simultaneous development of three streamers-CDS in the bubble and from the electrode tip, and ADS from the left side of the bubble. The maximum value is shown in each frame. Plots are on a log scale with the number of decades noted. 


\section{$\mathrm{P} / \mathrm{P}_{\mathrm{n}}=0.065$}

\section{$\mathrm{E} / \mathrm{N}(\mathrm{Td})$}

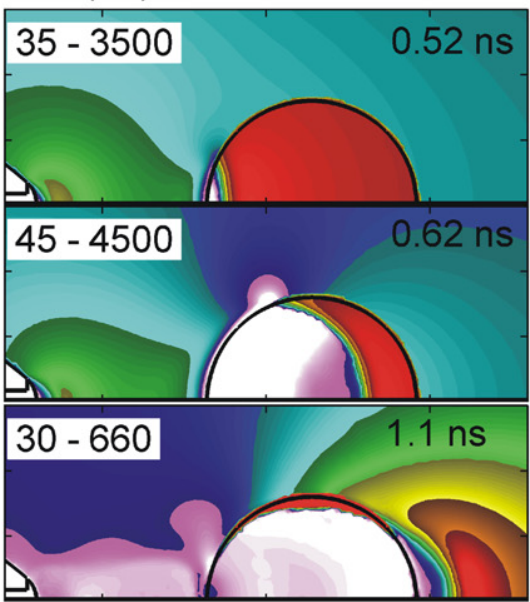

(a)
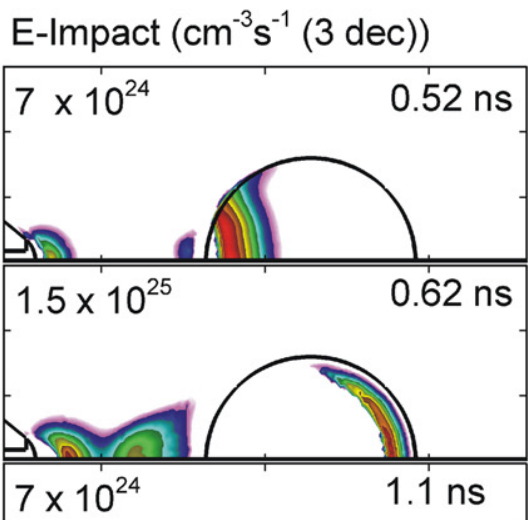

(b)
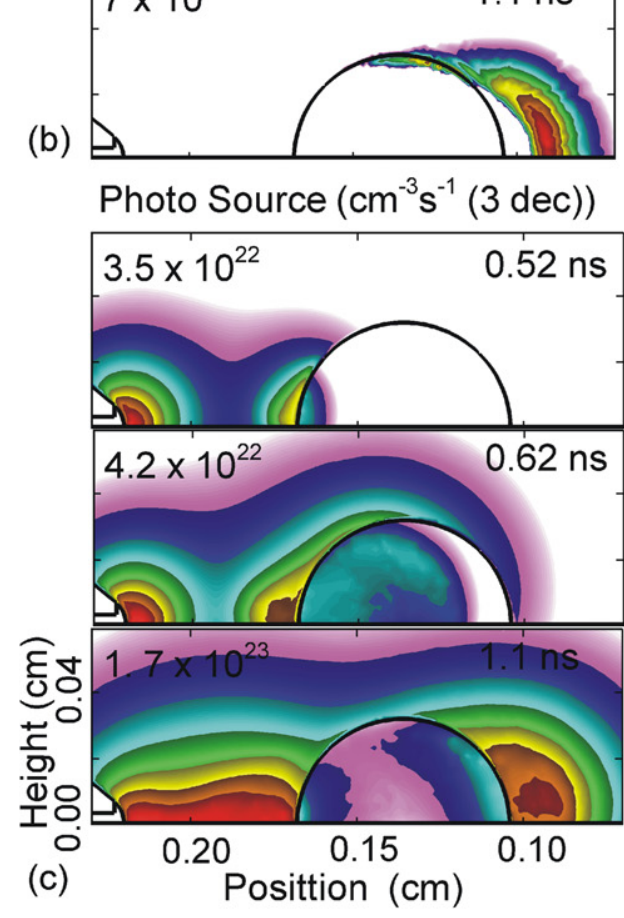

MIN

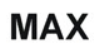

Figure 5. Streamer intersecting a single bubble of $320 \mu \mathrm{m}$ radius located on axis for ambient pressure of 3 atm and $P / P_{0}=0.065$. (a) Reduced electric field $E / N,(b)$ electron impact sources and (c) photoionization sources. The range of values plotted or maximum value is shown in each frame. Plots are on a log scale if number of decades is noted. the main positive streamer along the pre-ionized path. As the CDS from the electrode intersects the ADS from the bubble, the negative charge diminishes and the combined streamer takes on the characteristics of the CDS.

The positive CDS in the bubble crosses and passes through the bubble boundary on the cathode side and continues to propagate in the ambient, higher pressure gas. This CDS streamer has properties that are similar to those of a streamer in the absence of the bubble (e.g. $E / N$ of $600 \mathrm{Td}$ in the avalanche front). The plasma channel remaining in the bubble is confined by both ambipolar forces and the marked decrease in mobility that electrons and ions experience in crossing into the higher pressure ambient gas. The limits of the plasma channel are indicated by the positive charge at the boundary of the high mobility (bubble) and low mobility (ambient) regions. This accumulation of charge at the bubble surface has been experimentally seen in bubbles in liquids [23].

Recall that $T_{\mathrm{e}}$ is obtained by solving a continuum electron energy equation with transport coefficients given by solving Boltzmann's equation for the electron energy distribution (EED). Although the nonequilibrium and non-local nature of the $T_{\mathrm{e}}$ is captured using this method and, local kinetics are addressed by the locally varying EED, our treatment is strictly not kinetic. The computed $T_{\mathrm{e}}$ in the ionization wave exceeds $20 \mathrm{eV}$. This large value of $T_{\mathrm{e}}$ indicates that had a kinetic scheme been used, runaway electrons would likely have been produced. This stresses the importance of kinetic transport in these very high $E / N$ regions in bubbles.

The intersection of a streamer with single bubble $40 \mu \mathrm{m}$ in radius (smaller than the streamer width) with $P / P_{0}=0.065$ ( $P=150$ Torr, $P_{0}=3 \mathrm{~atm}$ ) is shown in figure 6 (electron density, net negative charge and net positive charge) and figure 7 ( $E / N$, electron impact sources and photoionization sources). The dynamics are similar to that of the larger bubble with the exception that the streamer narrows while approaching the bubble to be conformal to its size. As the CDS propagates from the electrode tip, photoionization seeds electrons inside the bubble which, with its large $E / N$, initiates both a CDS and an ADS. The larger separation between the electrode and the boundary of the smaller bubble makes more clear the distinction between the primary CDS streamer from the electrode tip and the ADS launched from the bubble. This larger separation and the longer propagation time allow the negative ADS from the bubble to independently propagate prior to intersecting with the primary CDS. On the other side of the bubble, the CDS launched from the bubble takes on essentially the same properties as a streamer in the absence of the bubble.

The narrowing of the primary streamer as it approaches the bubble can be explained, in part, by current continuity. The conductivity and current density in the bubble are higher than in the ambient medium. The electric field streamlines carrying current from the ambient into the bubble converge from a region of lower current density to that of a higher current density so that the total current is conserved.

\section{Streamer interaction with multiple bubbles}

The enhanced ionization occurring in bubbles and the ability to launch streamers from those bubbles may provide a means 


$$
\mathrm{P} / \mathrm{P}_{0}=0.065
$$

Electron density $\left(\mathrm{cm}^{-3}(3 \mathrm{dec})\right)$
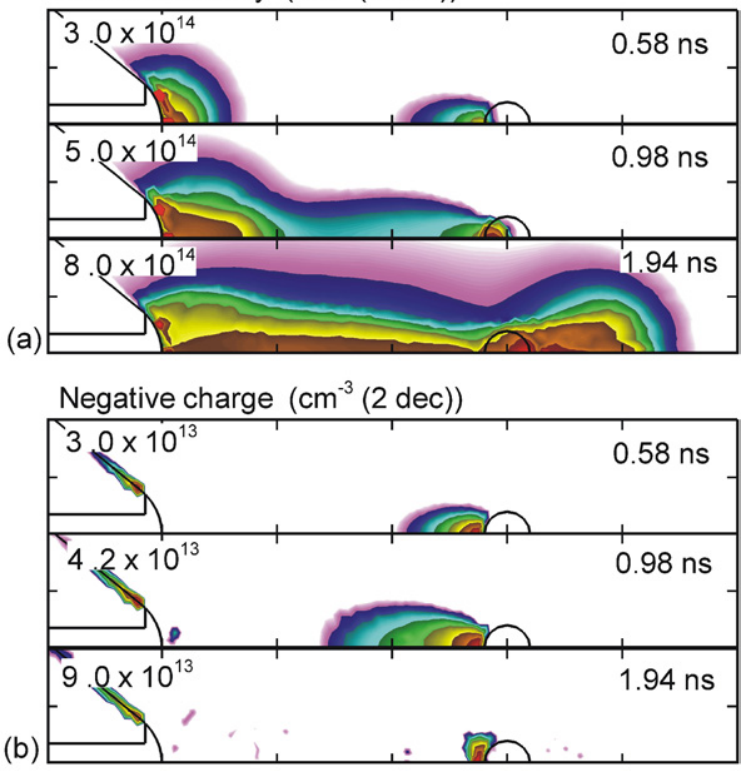

Positive charge $\left(\mathrm{cm}^{-3}(1 \mathrm{dec})\right)$

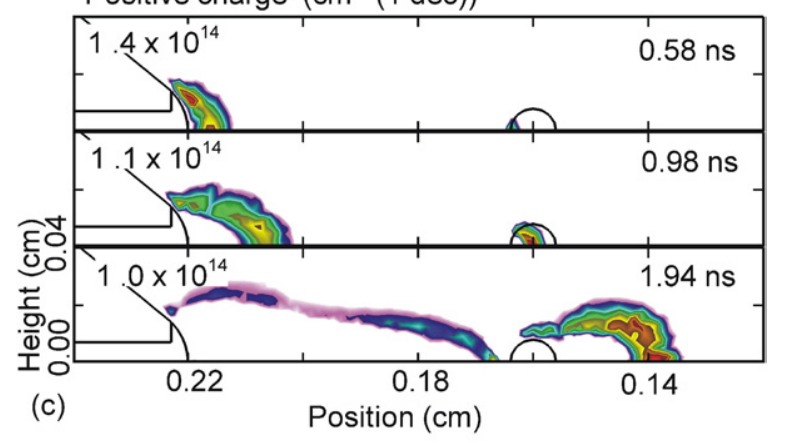

MIN

MAX

Figure 6. Streamer intersecting a single bubble of $40 \mu \mathrm{m}$ radius located on axis for ambient pressure of $3 \mathrm{~atm}$ and $P / P_{0}=0.065$. (a) Electron density, $(b)$ net negative charge and $(c)$ net positive charge. The dynamics are similar to that for the larger bubble with the exception that the streamer narrows while approaching the bubble to be conformal to its size. The range of values plotted or maximum value is shown in each frame. Plots are on a log scale if number of decades is noted.

for branching in dense media. Randomly occurring bubbles of different sizes in the vicinity of the primary streamer could be the sources of secondary streamers. In liquids the mean free paths are shorter for both the propagation of photoionizing radiation and for electrons. It is therefore more likely that secondary streamers would be launched in the immediate vicinity of the primary streamer, and not physically separated as we have seen in the prior section. The end result might be the appearance of branching. It is instructive to investigate the consequences of a streamer propagating in the vicinity of multiple, randomly placed bubbles in multi-atmosphere air as an upper-bound of what may occur in liquids.

To approximate the bubbles that might occur in a liquid, five bubbles with a radius of $40 \mu \mathrm{m}$ and pressure of 150 Torr were randomly placed near the electrode tip. The ambient pressure is $3 \mathrm{~atm}$ with 150 Torr in the bubbles $\left(P / P_{0}=0.065\right)$
$\mathrm{P} / \mathrm{P}_{0}=0.065$
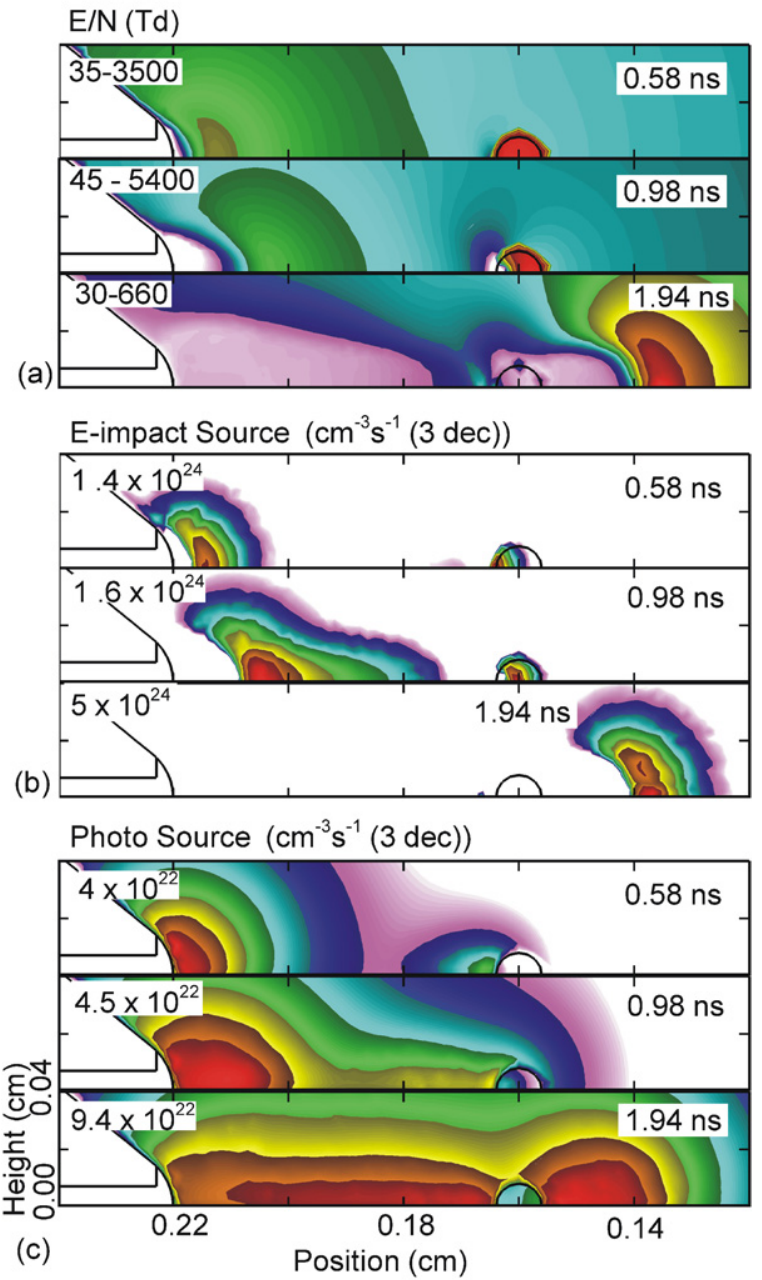

MIN

MAX

Figure 7. Streamer intersecting a single bubble of $40 \mu \mathrm{m}$ radius located on axis for ambient pressure of 3 atm and $P / P_{0}=0.065$. (a) Reduced electric field $E / N$, (b) electron impact ionization sources and $(c)$ photoionization sources. The range of values plotted or maximum value is shown in each frame. Plots are on a log scale if number of decades is noted.

providing a 15-to-1 density ratio. A preliminary report on simulations for a 5-to-1 density ratio is presented in [32]. The resulting plasma properties are shown in figure $8\left(E / N, T_{\mathrm{e}}\right)$, figures 9 (electron impact and photoionization sources) and 10 (positive charge and electron density).

A necessary process for the propagation of positive streamers is providing a source of ionization ahead of the ionization front. This source is typically photoionization produced by photons generated in the streamer. Photoionization produces electrons in front of the streamer which drift towards the anode into the large $E / N$ in the avalanche front, where they produce additional ionization thereby propagating the streamer towards the cathode. In principle, this ionization should result in both a CDS and an ADS. However, in a positive streamer the conductive channel behind the avalanche front is already formed and so the anode directed electrons produced in the avalanche front entering 


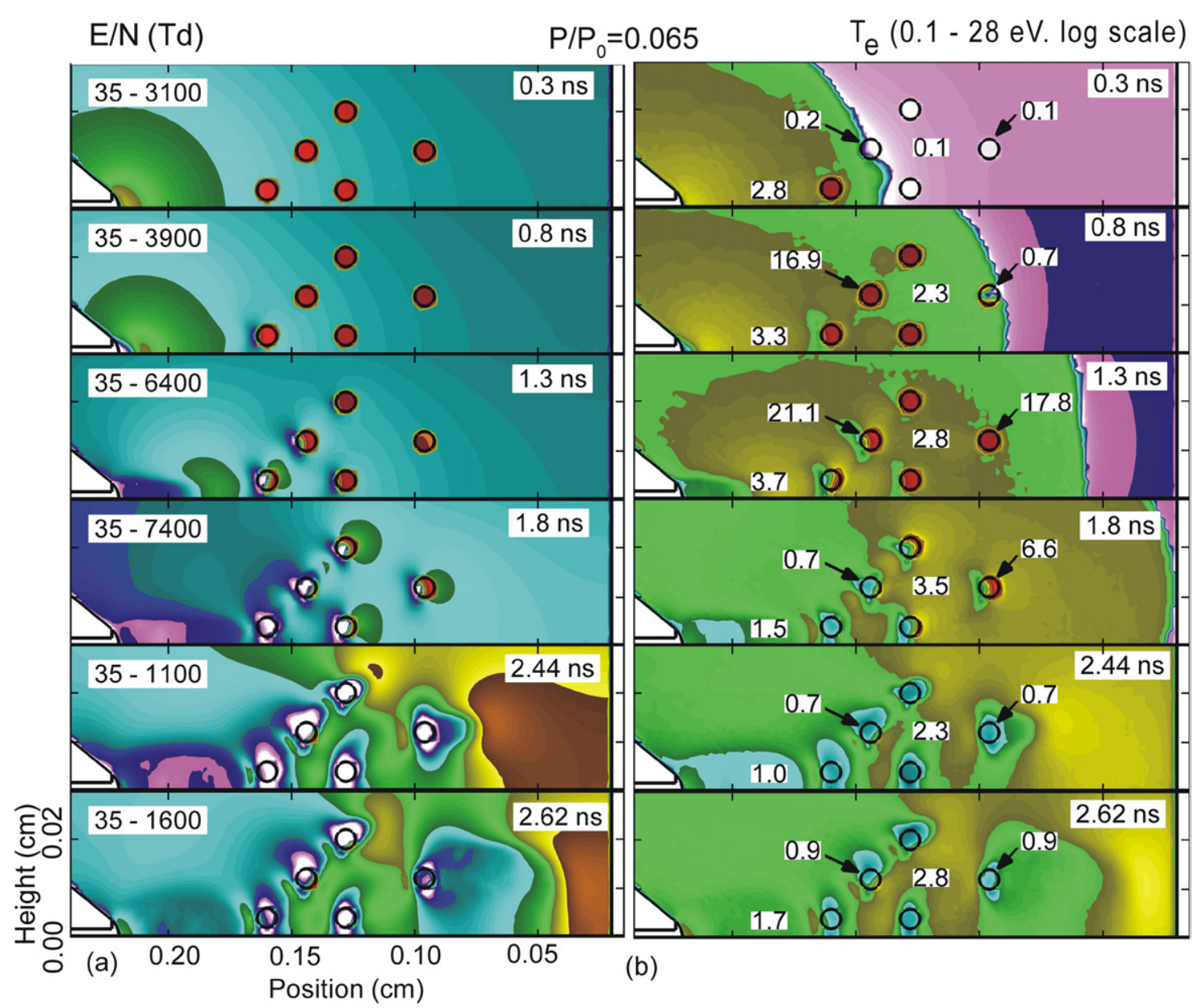

MIN

MAX

Figure 8. Plasma properties for streamer propagating through five randomly placed bubbles of $40 \mu \mathrm{m}$ radius in an ambient of $3 \mathrm{~atm}$. The 150 Torr pressure in the bubbles provides a rarefaction of $P / P_{0}=0.065$. (a) Reduced electric field $E / N$ and $(b)$ electron temperature $T_{\mathrm{e}}$. The initially large $E / N$ produces a correspondingly large $T_{\mathrm{e}}$, both of which collapse after avalanche occurs in the bubbles. Note that the low $T_{\mathrm{e}}$ in the bubbles when they do not yet contain electrons is not meaningful. The range of values plotted or maximum value is shown in each frame. Plots are on a log scale if number of decades is noted.

the conductive channel do little to change the course of the streamer.

When photoionization seeds electrons in the high $E / N$ of remotely located bubbles, there are no pre-existing conducting channels leading to either the cathode or anode from the bubbles. The avalanching electrons in the bubbles therefore are able to launch both a CDS and an ADS. As the primary (or secondary streamers) approach to within a few mean free paths of the bubbles for the ionizing radiation they produce, avalanches are launched from those streamers. Depending on their location and proximity to other streamers, the secondary streamer may either link up with another streamer or propagate independently.

This sequence of events is shown in figures $8(E / N$ and $T_{\mathrm{e}}$ ), 9 (electron impact ionization and photoionization sources) and 10 (positive charge and electron density). The $E / N$ in the bubbles is already larger than the ambient by the ratio of the pressures. As the large $E / N$ in the avalanche front approaches the bubbles (and voltage is compressed in front of the conductive channel), the $E / N$ in the bubble also increases coincident with the seeding of electrons by photoionization. $T_{\mathrm{e}}$ in the bubbles approaches $28 \mathrm{eV}$ (which should be interpreted as being in a runaway mode). These large $T_{\mathrm{e}}$ produce rates of ionization in excess of $10^{24} \mathrm{~cm}^{-3} \mathrm{~s}^{-1}$ which launch both a CDS and an ADS. The directions of the CDS are shown by the positive charge of their avalanche fronts and of their confining ambipolar fields. The avalanche within the bubbles quickly makes them more conductive than the surrounding gas, which lowers their $E / N$ below the ambient value. This also lowers $T_{\mathrm{e}}$ in the bubbles to $0.7-0.9 \mathrm{eV}$ which is also below ambient.

The arrangement and proximity of the bubbles ultimately determine the shape of the resulting streamer branches. In this example, the primary streamer initially propagates along the axis and intersects a bubble on axis. This bubble is in the proximity of two paths of bubbles leading approximately $45^{\circ}$ and $20^{\circ}$ off axis. The remotely seeded photoelectrons in these bubbles begin CDS and ADS, which in some cases link together to provide the appearance of branching. In this case, the primary streamer intersecting the first bubble stalls and does not propagate further along the axis. The streamers leaving 


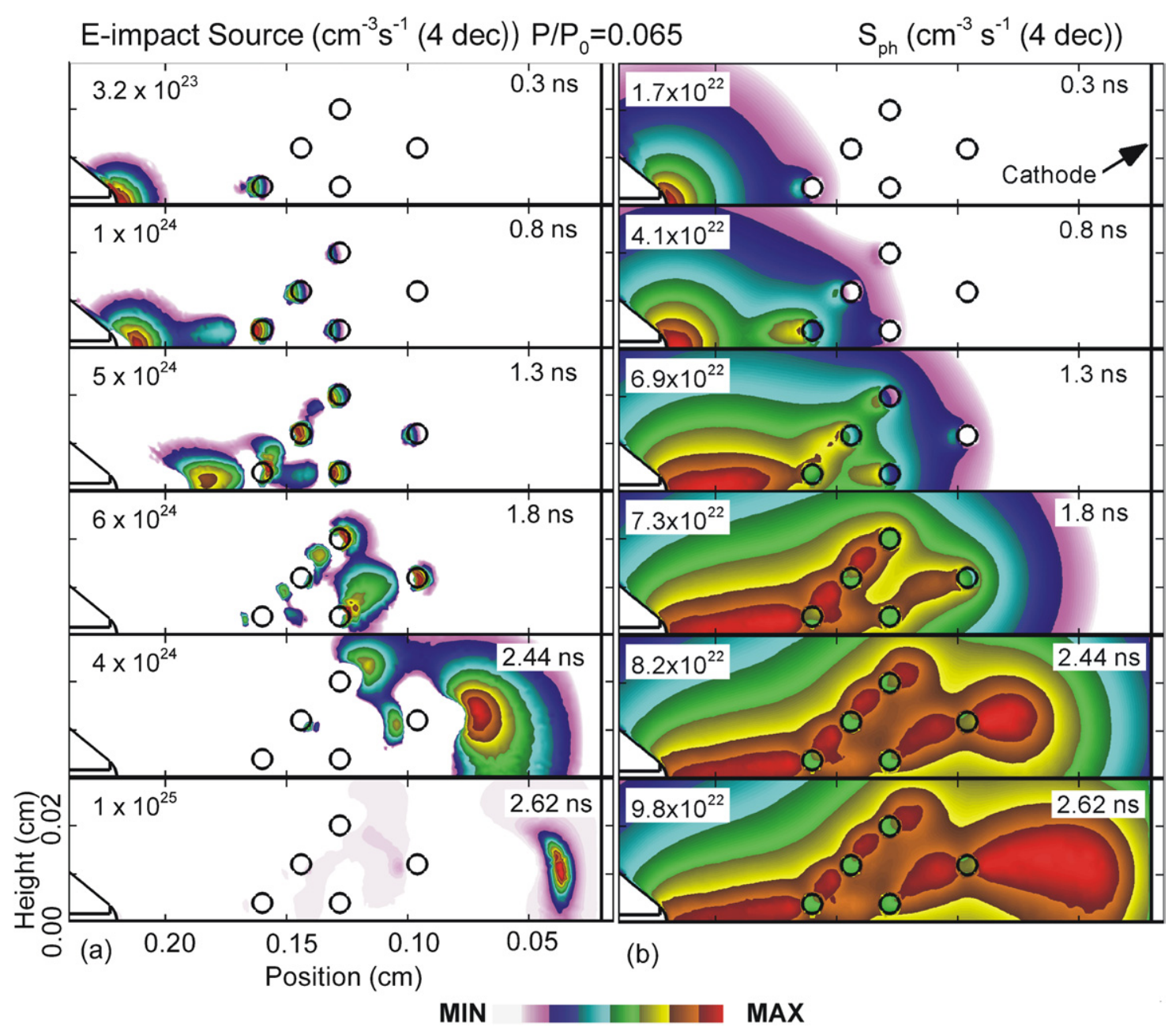

Figure 9. Plasma properties for streamer propagating through five randomly placed bubbles of $40 \mu \mathrm{m}$ radius for the conditions of figure 8 . (a) Electron impact ionization source and $(b)$ photoionization source. The ionization in the bubbles is only momentary prior to their becoming conductive. The maximum value plotted is shown in each frame. Plots are on a log scale with number of decades is noted.

the cathode sides of bubbles at the boundary of the bubbled region continue to propagate unimpeded as individual CDS. The ultimate fate of these streamers depends in part on where they were launched. In this example, the near-axis branch survives to propagate to the cathode. The branch further from the axis stalls and does not survive. The $E / N$ away from the axis is lower than on axis even in the absence of other streamers. However, the conductive channel produced by the near-axis branch of the streamer decreases the axial electric field at that height sufficiently to choke off ionization in the more remote branch.

The importance of the location and proximity of other bubbles in determining the survival of the streamers they launch is further illustrated by the electron density and $E / N$ shown in figure 11 for an ensemble of seven bubbles $40 \mu \mathrm{m}$ in radius randomly placed near the electrode tip. Early during the streamer development, the ambient $E / N$ is large enough that, with the amplification in $E / N$ in the bubbles, all of the bubbles avalanche and launch CDS and ADS. The upper bubbles are sufficiently far from the axis that their ADS do not intersect with the primary streamer prior to the primary streamer intersecting with bubbles near the axis. The plasma column of the on-axis streamer reduces $E / N$ at or above that height. The end result is that the streamers launched from the upper two bubbles die out without producing new branches. The primary streamer then splits into a small bush of streamers closer to the axis.

Small changes in the layout and number of bubbles have significant effects on the final branching pattern. For example, the electron density is shown in figure 12 for different numbers and arrangements of bubbles for $t=2.1 \mathrm{~ns}$ when all of the bubbles are already ionized. The streamer is most likely to follow paths of bubbles near the axis where the electric field and photoionization sources are largest. Closely spaced bubbles can, however, form a bridge that diverts the streamer off axis out of the region of largest electric field. The layouts for four bubbles differ only in the placement of a single bubble. Moving this bubble off axis creates a chain that diverts the streamer off axis. These observations are consistent with the experimental observations [1] that chains of bubbles in liquids can play the role of gaseous bridges along which a streamer may propagate.

\section{Bubble size, $P / P_{0}$ and Paschen's Curve}

Bubbles in liquids typically have a distribution of sizes. To investigate the role of bubble size for the branching, 


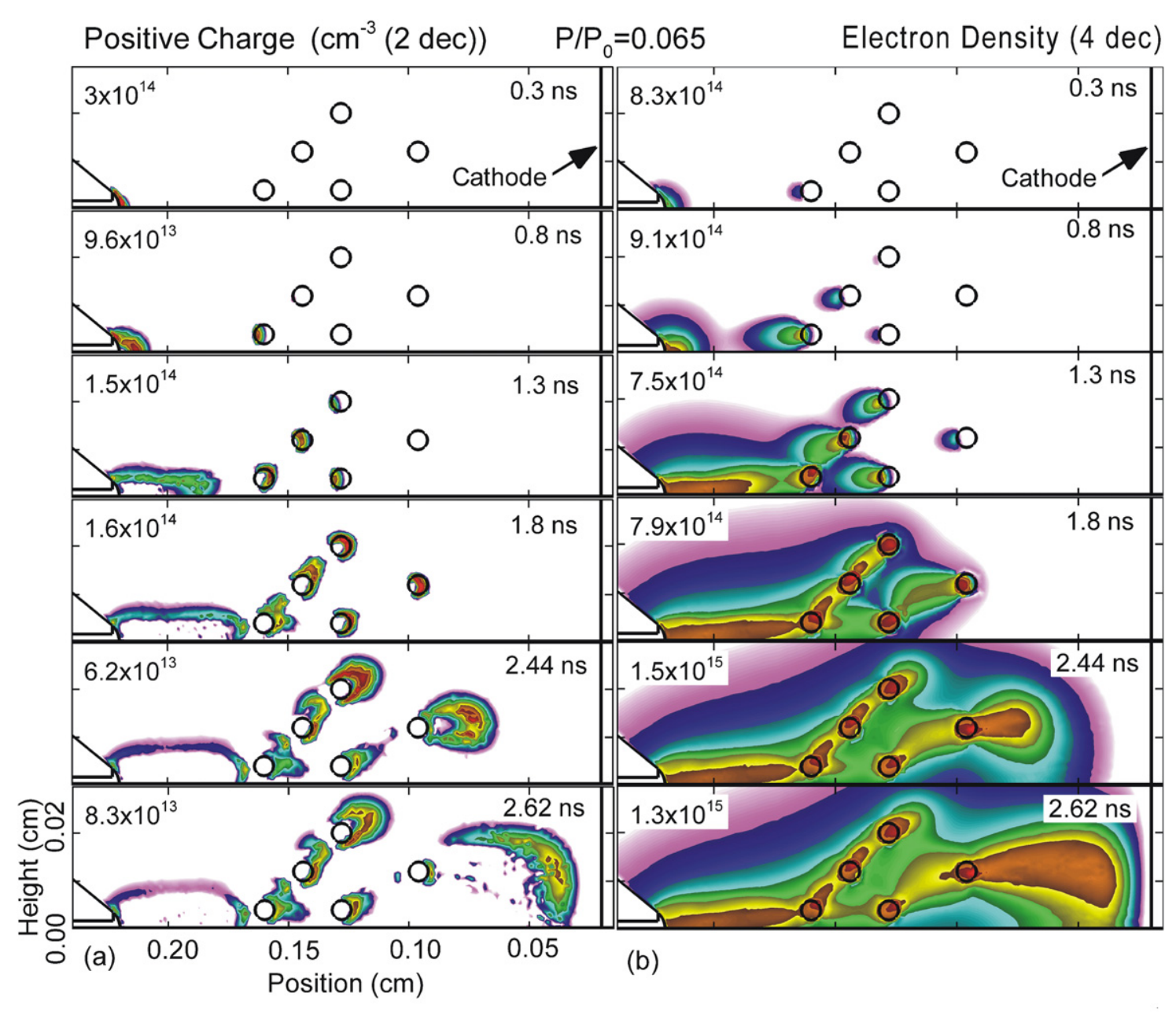

MIN

MAX

Figure 10. Plasma properties for streamer propagating through five randomly placed bubbles of $40 \mu \mathrm{m}$ radius for the conditions of figure 8 . (a) Net positive charge and $(b)$ electron density. The outline and direction of CDS are shown by the positive charge. The main streamer (starting from the anode) stalls in front of the ensemble of bubbles and splits into two branches One branch ultimately reaches the cathode while the other (located far from the axis in the less divergent electric fields) does not propagate. The maximum value plotted is shown in each frame. Plots are on a log scale with number of decades is noted.

we randomly placed an ensemble of five bubbles of radius $10 \mu \mathrm{m}$ with $P / P_{0}=0.065$ near the electrode tip, as shown in figure 13(a) (one bubble is to the right of the frame). The streamer branches from these small bubbles launch are narrower and comparable to their dimensions. As time progresses, these small branches stall with only the primary streamer propagating. The small amount of charge in the streamer heads is insufficient to create an avalanching value of $E / N$. The electron density for an ensemble of seven bubbles with the radii of $7-35 \mu \mathrm{m}$ is also shown in figure 13(a). The smallest bubble, even though placed near the axis where both $E / N$ and photoionization sources are larger, is not able to launch competitive streamers. Instead, the primary streamer takes a path following the larger bubbles located further off the axis which do critically launch streamers.

The value of $P / P_{0}$ in the bubbles is also important in determining their avalanche properties and contributions to possible branching. The electron density for an ensemble of seven bubbles of the same radius with different $P / P_{0}(0.065$, $0.2,0.33$ ) is shown in figure $13(b)$. Given that the ambient gas is at the same pressure and applied voltage is the same, the pressure inside the bubbles determines the $E / N$ inside the bubbles and so the speed of avalanche, if any. Smaller $P / P_{0}$ produces larger $E / N$, more rapid avalanche and more rapid development of branching. As $P / P_{0}$ increases towards unity, the primary streamer becomes more of a monofilament as in the absence of bubbles due to the lower rate of avalanche in the bubbles. Note that a decrease in $P / P_{0}$ in a bubble will reduce the production of seed electrons by photoionization by virtue of there being less absorption. This is partially offset by the higher value of $E / N$ which more rapidly avalanches those sparser electrons.

A possible explanation of the dependence of avalanche inside bubbles and launching of streamers from bubbles on their size and internal pressure is a Paschen curve like behavior. Paschen's curve specifies the minimum breakdown voltage for a gas based on the $p d$ (pressure $\times$ electrode gap) product. There is a low pressure branch requiring higher voltages for breakdown with decreasing pressure because electrons experience too few collisions. With increasing pressure, a minimum 


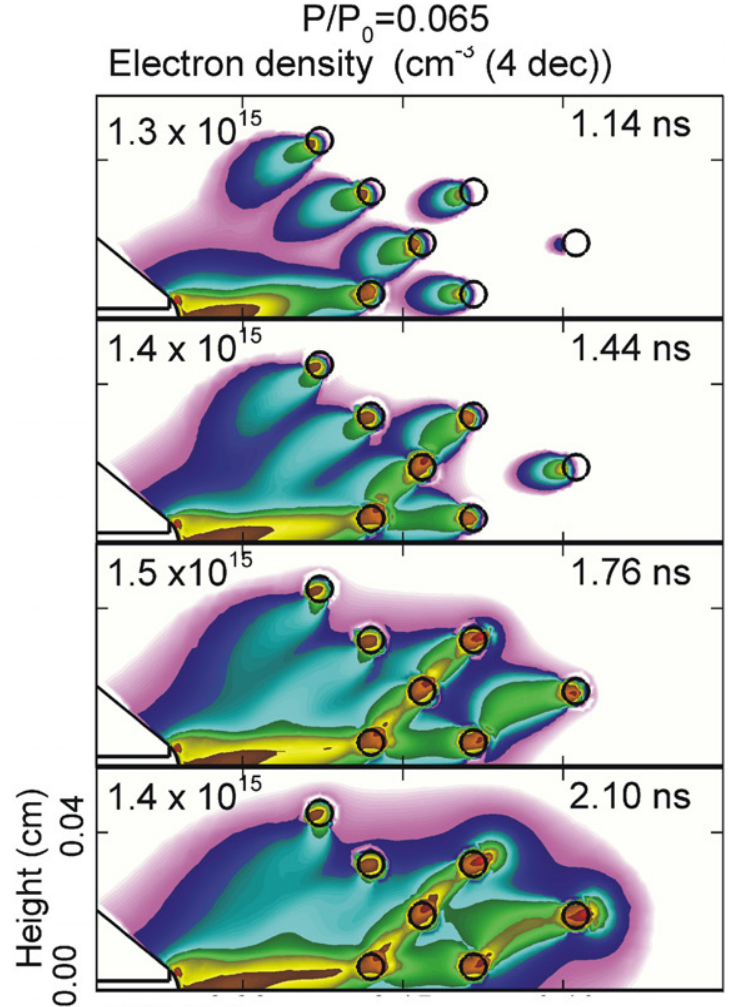

(a)

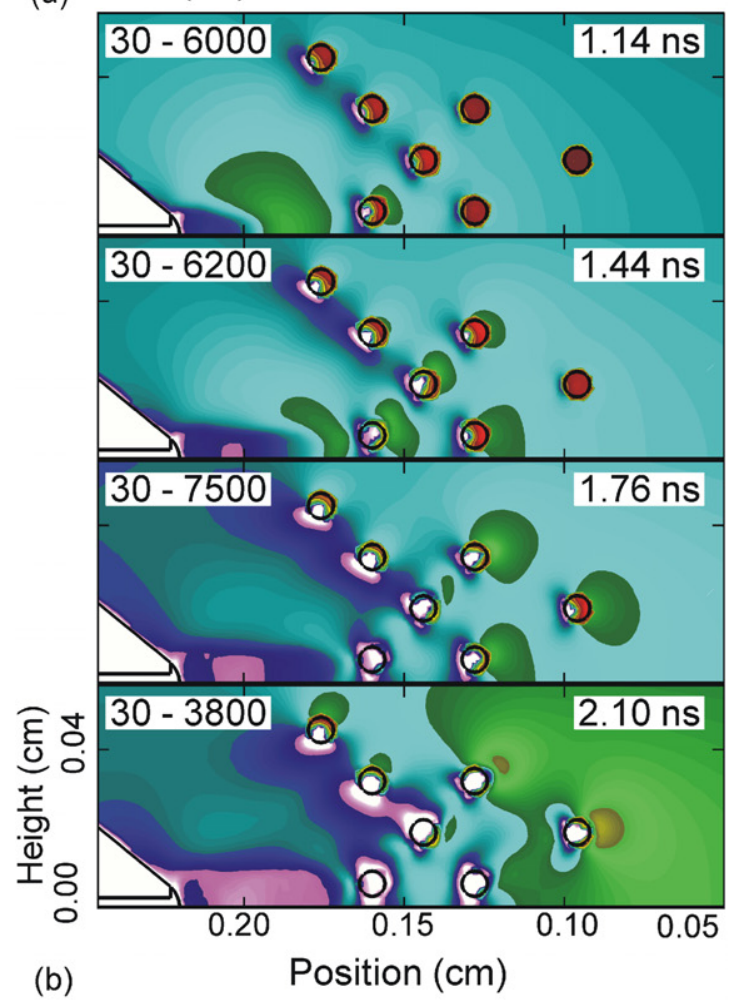

MIN

MAX

Figure 11. Streamer intersecting an ensemble of seven randomly distributed bubbles of $40 \mu \mathrm{m}$ radius with $P / P_{0}=0.065$. (a) Electron density and $(b)$ reduced electric field. The survival of streamer branches launched from bubbles depends in part on the $E / N$ in the ambient gas outside the streamer. The two upper bubbles initiate streamers which later die out as the near axis branches propagate. The range of values plotted or maximum value is shown in each frame. Plots are on a log scale if number of decades is noted.

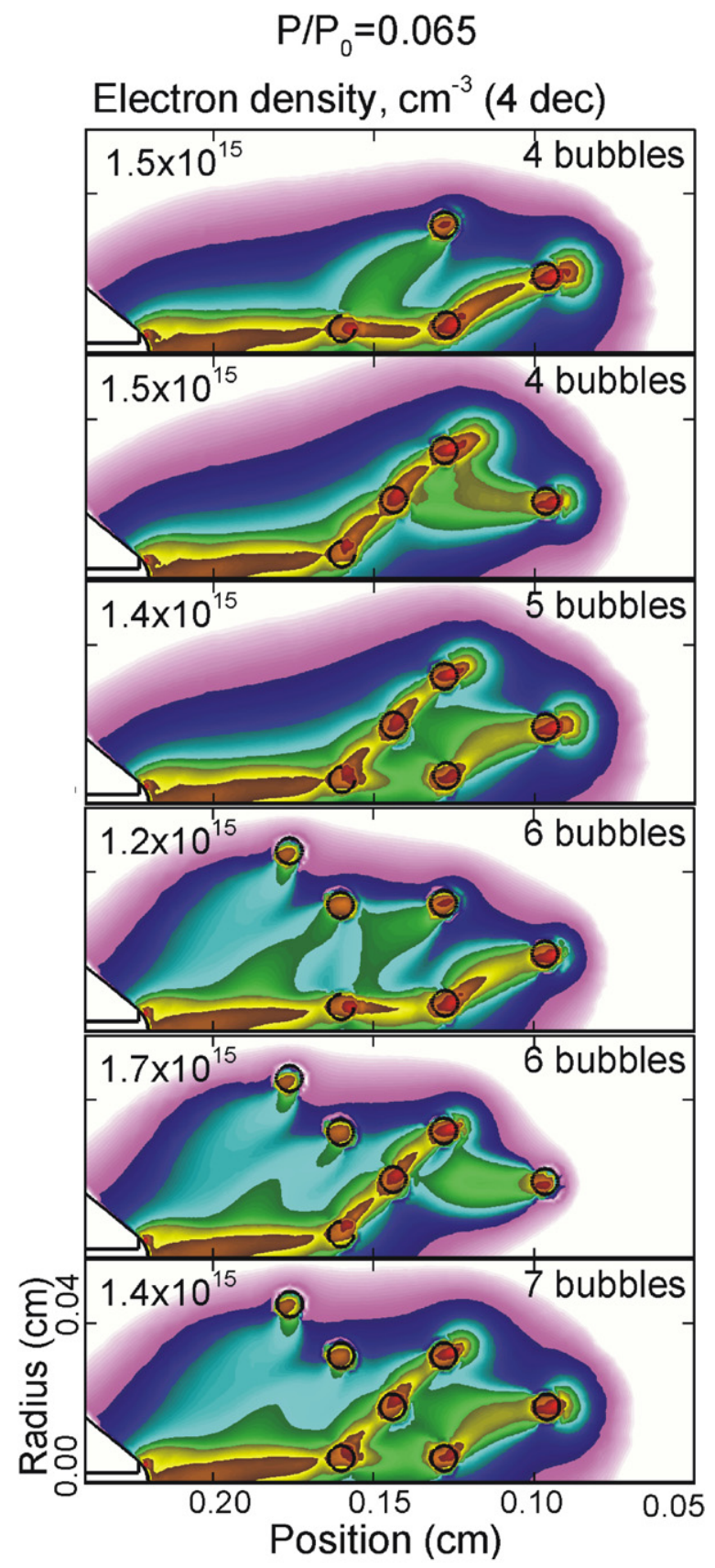

MIN

MAX

Figure 12. Electron density at $2.1 \mathrm{~ns}$ for streamers intersecting 4,5 , 6 and 7 bubbles $40 \mu \mathrm{m}$ in radius having different patterns ( $3 \mathrm{~atm}$, $\left.P / P_{0}=0.065\right)$. The branching pattern depends on the bubble positions. The propensity is for the streamer to propagate between closely spaced bubbles. The maximum value plotted is shown in each frame on a log scale.

is reached in breakdown voltage followed by a high pressure branch requiring higher voltages for breakdown (too many collisions). For air, the minimum occurs at $p d \approx 0.5$ Torr $\mathrm{cm}$ (or $6.6 \mathrm{~atm} \mu \mathrm{m}$ ) with a potential of about $350 \mathrm{~V} \mathrm{[29].}$

At the lower limit of bubbles investigated, a $7 \mu$ m radius bubble at 150 Torr has $p d=2.8 \mathrm{~atm} \mu \mathrm{m}$ which places it on the low side of the minimum of Paschen's curve for air. The largest voltage drop across the bubble for the conditions of figure 13 is only $115 \mathrm{~V}$, less than that required to self-avalanche in the 

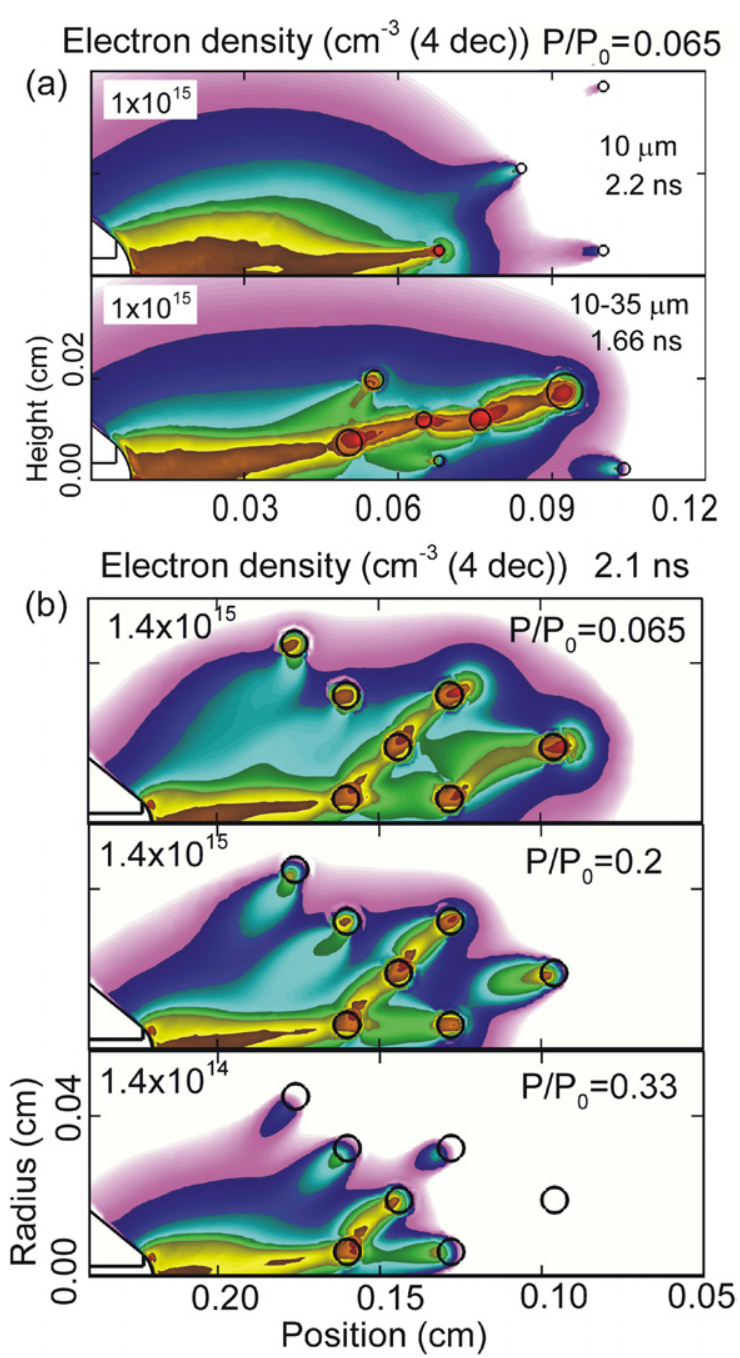

MIN

MAX

Figure 13. Electron density for different bubble properties. (a) Streamers for an ensemble of $10 \mu \mathrm{m}$ bubbles (upper frame), and bubbles of different radii 10 to $35 \mu \mathrm{m}$ (lower frame). Streamers launched from the small $10 \mu \mathrm{m}$ bubbles tend to stall. For bubbles of different sizes the streamer takes the path following larger bubbles located off axis. (b) Streamers intersecting bubbles of $40 \mu \mathrm{m}$ radius with $P / P_{0}=0.065,0.2$ and 0.33 . Bubbles are more likely to avalanche if their pd is located inside Paschen's curve. The maximum value plotted is shown in each frame on a log scale.

absence of external ionization. The $35 \mu \mathrm{m}$ bubble has $p d=$ $14 \mathrm{~atm} \mu \mathrm{m}$ which is on the high pressure side of the minimum of Paschen's curve. The bubble sustains a maximum potential drop of $575 \mathrm{~V}$, which is above the breakdown criterion. For the ensemble of bubbles shown in figure 13, we find that streamers develop and branch more rapidly for bubbles with a $p d$ value and voltage drop located inside Paschen's Curve. Less pronounced avalanche development occurs (if at all) for bubbles with $p d$ values outside of Paschen's curve.

\section{Mean free path of ionizing photons}

The mean free path of ionizing photons, $\lambda_{\mathrm{P}}$, plays an important role in streamer propagation and in initiating branching

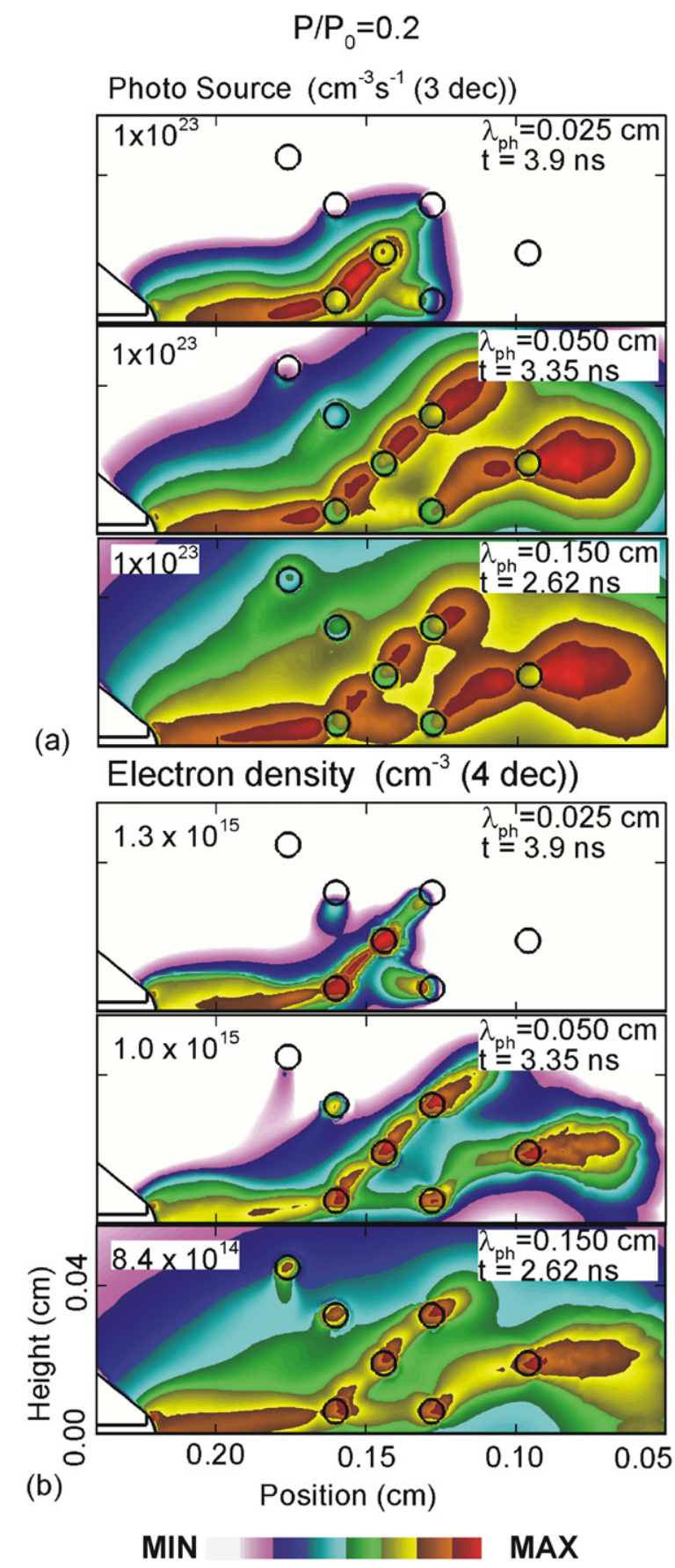

Figure 14. The role of mean free path $\lambda_{\mathrm{P}}$ of photons in streamer propagation. (a) Photoionization source and (b) electron density are shown for $\lambda_{\mathrm{P}}=0.025,0.050$ and $0.150 \mathrm{~cm}$. The maximum value plotted is shown in each frame on a log scale.

through bubbles. To some degree, $\lambda_{\mathrm{P}}$ determines the radius of the streamer channel and the speed of propagation [30,31]. The ability to launch streamers from bubbles and initiate branching depends on seeding of electrons in those bubbles by photoionization. As such, a small value of $\lambda_{P}$ not only narrows the width of the primary streamer and so make it less likely to intersect a bubble, but also reduces the likelihood that avalanches will occur in nearby bubbles.

For example, the photoionization source and electron density are shown in figure 14 for $\lambda_{\mathrm{P}}=250,500$ and $1500 \mu \mathrm{m}$. 
As the speed of propagation increases with increasing $\lambda_{\mathrm{P}}$, results are presented for progressively earlier times to show the streamers at similar stages of development. For small values of $\lambda_{\mathrm{P}}$, only the bubbles in close proximity to the streamer are seeded with electrons which, in the case of $\lambda_{\mathrm{P}}=250 \mu \mathrm{m}$, extends at most one bubble beyond the streamer. This is sufficient to enable the streamer to branch; however, $\lambda_{\mathrm{P}}$ is too small to propagate the streamer beyond the high electric field near the electrode tip, and the streamer stalls. With increasing $\lambda_{\mathrm{P}}$, bubbles further from the parent streamer are seeded with photoelectrons and avalanched, enabling more complex branching patterns, as in the case of $\lambda_{\mathrm{P}}=500 \mu \mathrm{m}$. For very large mean free paths, as for $\lambda_{\mathrm{P}}=1500 \mu \mathrm{m}$, there are abundant photoelectrons produced well beyond the bubbles, thereby broadening the streamer width sufficiently to envelope the bubbles and diminish their importance.

\section{Concluding remarks}

In this paper, we discussed the consequences of low pressure bubbles on the propagation of positive streamers in high pressure ( $3 \mathrm{~atm}$ ) humid air. The intent was to provide insights to how electrical discharges may propagate as streamers through liquids having bubbles. We found that the large $E / N$ in the bubbles resulting from their low density avalanches seed electrons produced by photoionization. Each bubble then potentially starts both negative and positive going streamers that may link with those from adjacent bubbles or the original streamer. When a streamer propagates through an ensemble of bubbles, the resulting avalanches appear as streamer branching. Bubbles in close proximity and in coincidental alignment are able to divert a streamer off axis from where the electric field is larger. The propensity for bubbles to avalanche depends on their $p d$ product and their placement on a Paschen-like curve for breakdown. Small bubbles with small $p d$ products may not avalanche due to there being too low a voltage drop across the bubble.

\section{References}

[1] Ushakov V Y, Klimkin V F and Korobeinikov S M 2007 Impulse Breakdown of Liquids (Berlin: Springer)

[2] Briels T M P, van Veldhuizen E M and Ebert U 2005 Trans. Plasma Sci. 33264
[3] Clements J S, Sato M and Davis R H 1987 Trans. Ind. Appl. IA-23 224

[4] Sunka P, Babicky V, Clupek M, Lukes P, Simek M, Schmidt J and Cernak M 1995 Plasma Source Sci. Technol. 8258

[5] Hoeben W, van Veldhuizen E M, Rutgers W R, Cramers C and Kroesen G 2000 Plasma Source Sci. Technol. 9361

[6] Sahni M and Locke B R 2006 J. Hazardous Mater. B 1371025

[7] Lukes P, Clupek M, Babicky V and Sunka P 2008 Plasma Sources Sci. Technol. 17024012

[8] Akiyama H 2000 Trans. Dielectr. Electr. Insul. 7646

[9] Sato M 2008 Plasma Sources Sci. Technol. 17024021

[10] Anpilov A M et al 2001 J. Phys. D: Appl. Phys. 34993

[11] Sun B, Kunitomo S and Igarashi C 2006 J. Phys. D: Appl. Phys. 393814

[12] Hara M, Wang Z-C and Saito H 1994 Trans. Dielectr. Electr. Insul. 1709

[13] Stalder K R, McMillen D F and Wolozko F 2005 J. Phys. D: Appl. Phys. 381728

[14] Denat A 2006 Trans. Dielectr. Electr. Insul. 13518

[15] Lukes P, Clupek M, Babicky V and Sunka P 2008 Trans. Plasma Sci. 361146

[16] Kanazawa S, Abe Y, Kihara Y, Kocik M and Mizeraczyk 2008 J. Trans. Plasma Sci. 36922

[17] Sarkisov G S, Zameroski N D and Woodworth J R 2006 J. Appl. Phys. 99083304

[18] An W, Baumung K and Bluhm H 2007 J. Appl. Phys. 101053302

[19] Kolb J F, Joshi R P, Xiao S and Schoenbach K H 2008 J. Phys. D: Appl. Phys. $\mathbf{4 1} 234007$

[20] Korobeinikov S M, Melekhov A V and Besov A S 2002 High Temp. 40652

[21] Korobeinikov S M, Melekhov A V Sinikh Yu N and Soloveichik Yu G 2001 High Temp. 39368

[22] Bruggeman P, Leys C and Vierendeels J 2007 J. Phys. D: Appl. Phys. 401937

[23] Gershman S, Mozgina S, Belkind A, Becker K and Kunhardt E 2007 Contrib. Plasma Phys. 4719

[24] Babaeva N Yu and Naidis G V 1999 Tech. Phys. Lett. 2591

[25] Qian J et al 2005 J. Appl. Phys. 97113304

[26] Babaeva N Yu and Kushner M J 2009 Plasma Sources Sci. Technol. 18035009

[27] Babaeva N Yu and Naidis G V 1996 J. Phys. D: Appl. Phys. 292423

[28] Pancheshnyi S, Nudnova M and Starikovskii A 2005 Phys. Rev. E 71016407

[29] Paschen F 1889 Ann. Phys., Lpz. 27369

[30] Naidis G V 2006 Plasma Sources Sci Technol. 15253

[31] Nudnova M M and Starikovskii A Yu 2008 J. Phys. D: Appl. Phys. 41234003

[32] Babaeva N Yu and Kushner M J 2008 Trans. Plasma Sci. 36892 